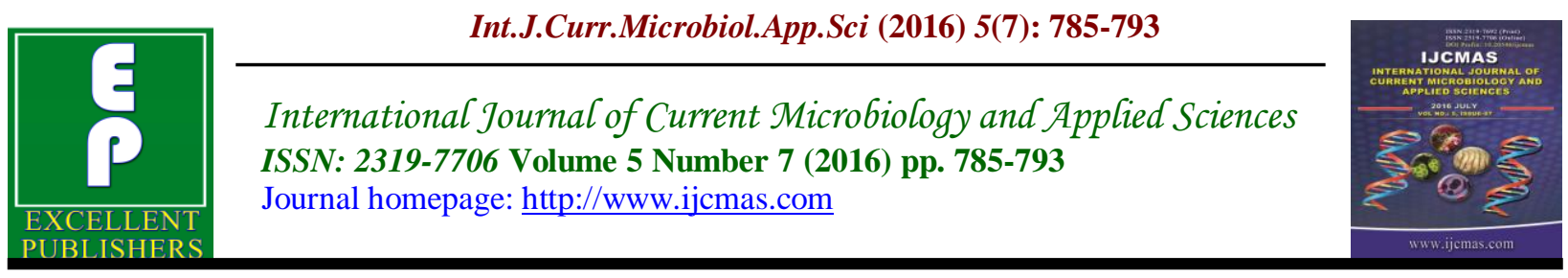

Original Research Article

http://dx.doi.org/10.20546/ijcmas.2016.507.090

\title{
Bactericidal Application of Biosynthesized Silver Nanoparticles
}

\author{
Hala Ezzat Abou El-Hassayeb* \\ Department of Marine Microbiology, National Institute of Oceanography \\ and Fisheries, Alexandria, Egypt \\ *Corresponding author
}

\begin{abstract}
A B S T R A C T
\section{Keywords}

Pseudomonas aeruginosa,

Biosynthesis, Silver nanoparticles,

Nitrate reductase, antimicrobial activity.

\section{Article Info}

Accepted:

25 June 2016

Available Online:

10 July 2016

Silver bionanoparticles have been known to have inhibitory and bacterial effects. Apart from standardizing the best parameter for the synthesis of silver nanoparticles involved in reduction of silver ion to silver nanoparticles. The involvement of nitrate reductases as reducing agent was confirmed by biochemical assay. The nitrate reductase activity got reduced from $0.9876 \mathrm{mmole} / \mathrm{min} / \mathrm{ml}$ to $0.3233 \mathrm{mmole} / \mathrm{min} / \mathrm{ml}$ after biofabrication of silver nanoparticles. In the present study, Pseudomonas aeruginosa ATCC 9027 has been used for the biosynthesis of silver nanoparticles (SNPs). It was found that aqueous $\mathrm{Ag}^{2}$ ions in solution when exposed to Pseudomonas aeruginosa ATCC 9027 get reduced, thereby leading to the formation of silver nanoparticles. The formation of silver nanoparticles was confirmed by the change in colour of the culture filtrate from yellow to brown after the addition of silver nitrate. The morphology and uniformity of silver nanoparticles were investigated by UV-Vis spectrum, X-ray diffraction and scanning electron microscope (TEM). These biosynthesized silver nanoparticles were also evaluated for their antimicrobial activity against Pseudomonas aeruginosa ATCC 9027, Staphylococcus aureus ATCC 2592 and Escherichia coli ATCC 8739. It was interesting to note that Pseudomonas aeruginosa ATCC 9027 that biosynthesized the silver nanoparticle was most affected by its antibacterial activity applications.
\end{abstract}

\section{Introduction}

Nowadays, nanotechnology has been developing rapidly as an important field of modern research with potential effects in medicine, agriculture, textiles, electronics, and energy, etc. Nanotechnology involves the development of efficient systems at the molecular scale. Nanotechnology is responsible for the synthesis and design of structures ranging in size between 1 and $100 \mathrm{~nm}$ (Rai et al., 2014). The synthesis of silver nanoparticles is an important aspect of nanotechnology. Presently, different techniques are available for the synthesis of silver nanoparticles, such as: chemical, physical, and biological. In the past decade, a great attention has been paid to microbial production of nanoparticles. Biosynthesis of silver nanoparticles (AgNPs) is an ecofriendly approach by using different biological sources; for example, plants and microorganisms such as bacteria, fungi, and actinobacteria (Tsibakhashvili et al., 2011; v 
et al., 2013; Gupta et al., 2014; Rai et al., 2015). Outbreak of the infectious diseases is caused by different pathogenic bacteria and the development of antibiotic resistance the pharmaceutical companies and the researchers are searching for new antibacterial agents. In the present scenario, nanoscale materials have emerged up as novel antimicrobial agents owing to their high surface area to volume ratio and the unique chemical and physical properties Nanotechnology refers broadly to a field of applied science and technology whose unifying theme is the control of matter on the atomic and molecular scale.

The metal microbe interactions have an important role in several biotechnological applications including the fields of bioremediation, biomineralization, bioleaching, and microbial corrosion (Bruins et al., 2000. Recently a few microorganisms have been explored as potential biofactories for synthesis of metallic nanoparticles such as cadmium sulfide, gold, and silver (Sastry et al., 2003; Tillmann, 2004; Ahmad et al., 2005; Shah et al., 2012).Research in nanotechnology provides reliable, ecofriendly processes for the synthesis of nanoscale materials. Inspiration from nature comes through magnetotactic bacteria synthesizing magnetite nanoparticles, diatoms synthesizing siliceous materials and S-layer bacteria producing gypsum and calcium carbonate layers. Marcetol (Duran et al., 2003) showed that silver nanoparticles (SNPs), like their bulk counterpart, are an effective antimicrobial agent against various pathogenic microorganisms.

The present work has focused on the development of an extracellular biosynthesis of SNPs using Pseudomonas aeruginosa and characterization of these nanoparticles have been carried out by UV-Vis Spectroscopy, XRD and TEM techniques. Moreover, in vitro antimicrobial activity of AgNPs synthesized from Pseudomonas aeruginosa was evaluated against Pseudomonas aeruginosa ATCC 9027, Staphylococcus aureus ATCC 2592 and Escherichia coli ATCC 8739.

\section{Materials and Methods}

\section{Culturing the microbe}

The bacterium Pseudomonas aeruginosa ATCC 9027 (Saitou et al., 1987) was obtained from Marine Microbiology department, National Institute of oceanography and fisheries, Alexandria, Egypt (NIOF). A loopful of Pseudomonas aeruginosa culture was inoculated in $250 \mathrm{ml}$ conical flask containing $100 \mathrm{ml}$ sterile Nutrient Broth. The inoculated medium was incubated at $37^{\circ} \mathrm{C}$ in a rotary shaker at 120 rpm for 24 hours. After 24 hours, the culture was centrifuged to separate bacterial cells. Centrifugation was done at $5000 \mathrm{rpm}$ for 10 minutes. Supernatant and pellet were separated. The supernatant obtained after centrifugation was used for nanoparticles synthesis.

\section{Synthesis of Nanoparticles}

Nutrient broth was prepared, sterilized and incubated with a fresh growth of test strain Pseudomonas aeruginosa ATCC 9027. The cultured flasks at $150 \mathrm{rpm}$. After the incubation period, the culture was centrifuged at $12,000 \mathrm{rpm}$ for10 min and the supernatant was used for the synthesis of silver nanoparticles (AgNPs). The supernatant of Pseudomonas aeruginosa ATCC 9027 culture was separately added to the reaction vessels containing silver nitrate at a concentration of $0.1 \mathrm{~g} / \mathrm{L}$.

The reaction between these supernatant and silver ions was carried out in bright conditions for $72 \mathrm{~h}$. The bioreduction of the silver ions in the solution was monitored by sampling the aqueous solution $(2 \mathrm{~mL})$ and 
measuring the absorption spectrum of the solution using (Beckman-Du-50) UVVisible spectrophoto-meter at a resolution of $1 \mathrm{~nm}$.

\section{Characterization of silver nanoparticles (AgNPs)}

\section{Visual detection}

After $4 \mathrm{~h}$ of incubation, the preliminary detection of silver nanoparticles was carried out by visual observation of colour change from yellow to brown. The change in colour clearly indicates the formation of AgNPs.

\section{UV-Vis Measurements}

The optical density of these nanoparticles suspended in distilled water was measured by UV-Visible spectrophotometer (Systronics 2202 double beam model) from wavelengths 200-700nm.. The SPR peaks were assessed for size and distribution of Silver nanoparticles.

\section{Transmission Electron Microscopic, TEM Examination of the Nanoparticle}

A scanning electron microscope (HRTEM) was used to record the micrograph images of synthesized silver nanoparticles.

\section{XRD Measurements}

The formation and quality of nanoparticles were checked by XRD technique. X-ray Diffraction (XRD) measurements of dropcoated films of synthesized nanoparticles on glass substrate were recorded in a wide range of Bragg angles $2 \theta$ at a scanning rate of 20min-1, carried out on a Philips PW 1830 instrument that was operated at a voltage of $40 \mathrm{kV}$ and a current of $30 \mathrm{~mA}$ with $\mathrm{Cu} \mathrm{K} \alpha$ radiation $(\lambda=1.5405 \AA)$.

\section{Elimination of untreated $\mathrm{Ag}^{+}$ions}

To eliminate the unreacted $\mathrm{Ag}^{+}$from the nanoparticle suspension, the reaction sample was treated with $1 \% \mathrm{NaCl}$ solution. The precipitate of $\mathrm{AgC} 1$ was removed by centrifugation at 4,500 $\mathrm{g}$ for $15 \mathrm{~min}$ followed by filtration with Whatman filter paper No. 1. Then the AgNPs were centrifuged at $11,000 \mathrm{~g}$ for $30 \mathrm{~min}$ and dried at $50^{\circ} \mathrm{C}$. The dried powder of AgNPs was used for antibacterial activity $\left(200 \mu \mathrm{g} \mathrm{ml}^{-1}\right)$ and minimum inhibitory concentration (MIC) in a concentration range of $5-200 \mu \mathrm{g} \mathrm{ml}^{-1}$ after dissolving in deionized water.

\section{Nitrate Reductase Assay}

For extraction of Nitrate Reductase from Pseudomonas aeruginosa, the supernatant obtained from the above procedure was homogenized with Tris- $\mathrm{HCl}$ buffer $(\mathrm{pH} 8.0)$ and then centrifuged at $2000 \mathrm{rpm}$ for 15 min. The supernatant was used as enzyme source. Nitrate Reductase activity was measured by Vega and Cardenas method. The standard graph was calibrated using 50 $\mu \mathrm{M}$ working standard of Sodium nitrite. To $0.1 \mathrm{ml}$ supernatant known amount of $0.1 \mathrm{M}$ $\mathrm{KNO}_{3}$ was added and incubated for 24 hours. Then $1 \mathrm{ml}$ of diazo coupling reagent (1\% Sulphanilamide in $3 \mathrm{ml} \mathrm{HCl}$ and $0.02 \%$ $\mathrm{N}$-(1-naphthyl) ethylenediamine hydrochloride) was added to $3 \mathrm{ml}$ reaction mixture and diluted 10 folds to detect the remaining $\mathrm{NO}_{2}$. After $30 \mathrm{~min}$ of incubation in dark at $37^{\circ} \mathrm{C}$ for development of color; O.D. was recorded at $540 \mathrm{~nm}$. The result was calculated against the standard graph of nitrite.

Determination of antimicrobial activity by well-diffusion method

The SNPs synthesized from Pseudomonas aeruginosa ATCC 9027 were tested for 
antimicrobial activity by well-diffusion method against pathogenic organisms such as Escherichia coli ATCC 8739, Staphylococcus aureus ATCC 2592 and Pseudomonas aeruginosa ATCC 9027. The pure cultures of organisms were subcultured on Nutrient broth at $37^{\circ} \mathrm{C}$ on a rotary shaker at $200 \mathrm{rpm}$. Wells of $6-\mathrm{mm}$ diameter were made on Nutrient agar plates using gel puncture. Each strain was swabbed uniformly onto the individual plates using sterile cotton swabs. Using a micropipette, $20 \mu \mathrm{L} \quad(0.002 \mathrm{mg})$ of the sample of nanoparticles solution was poured onto each of two wells and the third one as a control on all plates. After incubation at $37^{\circ} \mathrm{C}$ for 18 hours, the different levels of zone of inhibition were measured.

\section{Results and Discussion}

\section{Characterization of Synthesized Silver Nanoparticles (AgNPs)}

In the present study, the biosynthesis of silver nanoparticles by Pseudomonas aeruginosa ATCC 9027 was studied. Visual observations showed a change of colour in silver nitrate solution from yellow to brown( Fig. 1), whereas no colour change was observed in the culture supernatant without silver nitrate or in media with silver nitrate alone. The appearance of of yellowish brown colour in silver nitrate treated culture supernatant suggested the formation of silver nanoparticles (Sastry et al., 2003; Vighneshwaan et al., 2000; Konish et al., 2004).

The synthesized AgNPs were characterized by UV-Vis spectroscopy (Tillmann et al., 2004). In the UV-Vis absorption spectrum, the absorbance scan taken by UV-VIS spectrophotometer showed a sharp plasmon peak at $\sim 435 \mathrm{~nm}$ confirming the presence of silver (Figure 2). Observation of this peak, assigned to a surface Plasmon, is well documented for various metal nanoparticles with sizes ranging from 2-100 $\mathrm{nm}$.

$\mathrm{X}$ - ray diffraction (XRD) was carried out to confirm the crystalline nature of the particles and the XRD pattern obtained is shown in Figure 3. A comparison of the XRD data with the standard (joint committee in powder diffraction standards file no:040783)confirmed that the particles formed in our experiments were silver nanocrystals which can be depicted by the peaks at $2 \theta$ values of $38.45,44.48,64,69$ and 77.62 corresponding to (111), (200), (220), and (311) planes for silver respectively. This XRD pattern confirms the crystallinity of SNPs. The mean particle diameters of SNP were calculated from the XRD data which can be derived by Debye Scherrer equation.

$\mathrm{D}=\mathrm{K} \lambda / \beta^{1 / 2} \cos \theta$

This equation exploits the reference peak width at angle $\theta$, where $\lambda$ is the $x$-ray wavelength (1.5418), $\beta 1 / 2$ is the width of the $\mathrm{XRD}$ peak at half height and $\mathrm{K}$ is the shape factor. The calculated average particle size of SNP was 20-50 nm which can also be confirmed by TEM results.

Transmission electron microscopy (TEM) images of nanoparticles that were synthesized by Pseudomonas aeruginosa indicated that the nanoparticles were in the size range of 10 to $20 \mathrm{~nm}$ (Figure 4 ) which is in close agreement with the particle size calculated from the XRD profile.

\section{Nitrate Reductase Assay}

Studies have indicated that $\mathrm{NADH}$ and $\mathrm{NADH}$-dependent nitrate reductase enzyme are important factors in the biosynthesis of metal nanoparticles. Pseudomonas aeruginosa is known to secrete the cofactor $\mathrm{NADH}$ and NADH-dependent enzymes, 
especially nitrate reductase, which may be acting as a scaffold or nucleating agent and might be responsible for the bioreduction of $\mathrm{Ag}+$ to $\mathrm{Ag}^{0}$ and the subsequent formation of Silver nanoparticles. The same enzyme later then acts as a capping agent, thus ensuring complete formation of thermodynamically stable nanostructures. The molecular activity of nitrate reductase in the bacterial exudates of P.aeruginosa was found to be 0.9876 $\mathrm{mmole} / \mathrm{min} / \mathrm{ml}$; which got reduced to 0.6546 $\mathrm{mmole} / \mathrm{min} / \mathrm{ml}$ when it was subjected to $100^{\circ} \mathrm{C}$ (Fig 5). After the formation of silver nanoparticles the nitrate reductase activity was again assayed in the reactant mixture which showed a substantial decrease with $0.3233 \mu \mathrm{mole} / \mathrm{min} / \mathrm{ml}$ in the solutions (bacterial exudates) having Silver nanoparticles as compared to nitrate reductase activity in bacterial exudates without silver nanoparticles. This result confirms the involvement of nitrate reductase in the reduction of silver ion to silver nano particles. The fact that which capping proteins are involved in stabilizing the particle is yet to be explored.

\section{Anti-microbial assay}

The antibiotic activity of SNPs was investigated against various pathogenic organisms such as Escherichia coli, Staphylococcus aureus and Pseudomonas aeruginosa using well-diffusion method (Fig $6)$. The mean of three replicates of the diameter of inhibition zones (in millimeters) around each well with SNPs solution is represented in (Table1). The highest antimicrobial activity was observed against Pseudomonas aeruginosa followed by Staphylococcus aureus and Escherichia coli.

Table.1 Zone of inhibition of SNPs against various pathogenic bacteria Pathogenic bacteria Diameter of Zone of inhibition (mean of 4 replicates)

\begin{tabular}{|c|c|}
\hline Pathogenic bacteria & Diameters of zone of inhibition (mean of 3 replicates) \\
\hline Pseudomonas aeruginosa & $24 \mathrm{~mm}$ \\
\hline Staphylococcus aureus & $18 \mathrm{~mm}$ \\
\hline Escherichia coli & $15 \mathrm{~mm}$ \\
\hline
\end{tabular}

Fig.1 Conical flasks containing Pseudomonas aeruginosa culture supernatant in aqueous AgNO3 solution: (A) At the beginning of reaction showing no colour change; \&(B) After $72 \mathrm{~h}$ of reaction showing brown colour.

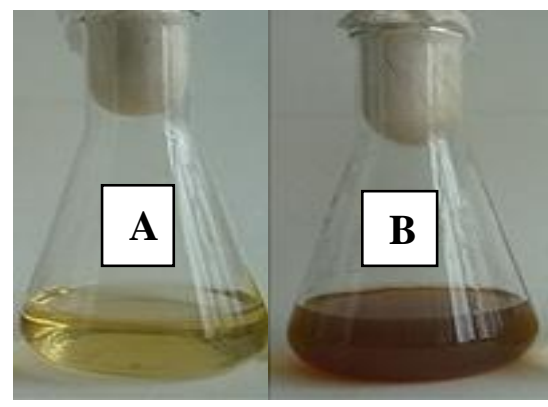


Fig.2 UV-Vis absorbance of AgNPs

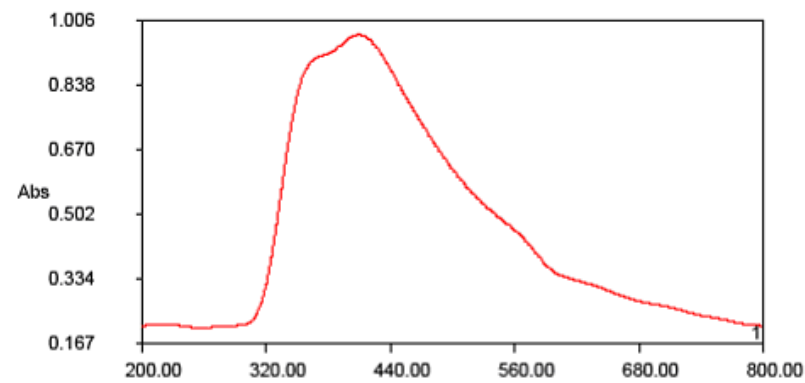

Fig.3 XRD pattern of silver nano particles synthesized using bacterial exudates of Pseudomonas aeruginosa

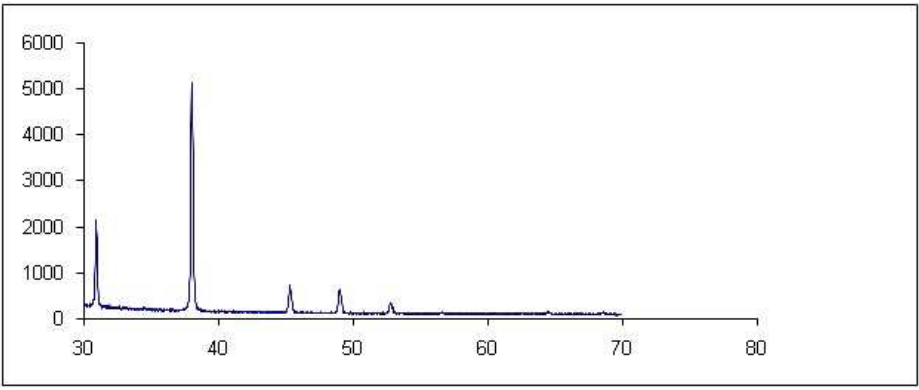

Fig.4 TEM image of Silver nanoparticles synthesized using Pseudomonas aeruginosa

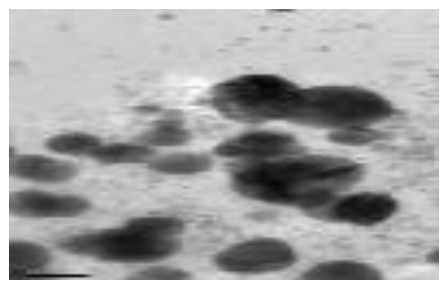

Fig.5 Nitrate reductase activity of Pseudomonas aeruginosa exudates, Boiled bacterial exudates and silver nanoparticles respectively in $\mu$ moles $/ \mathrm{min} / \mathrm{ml}$

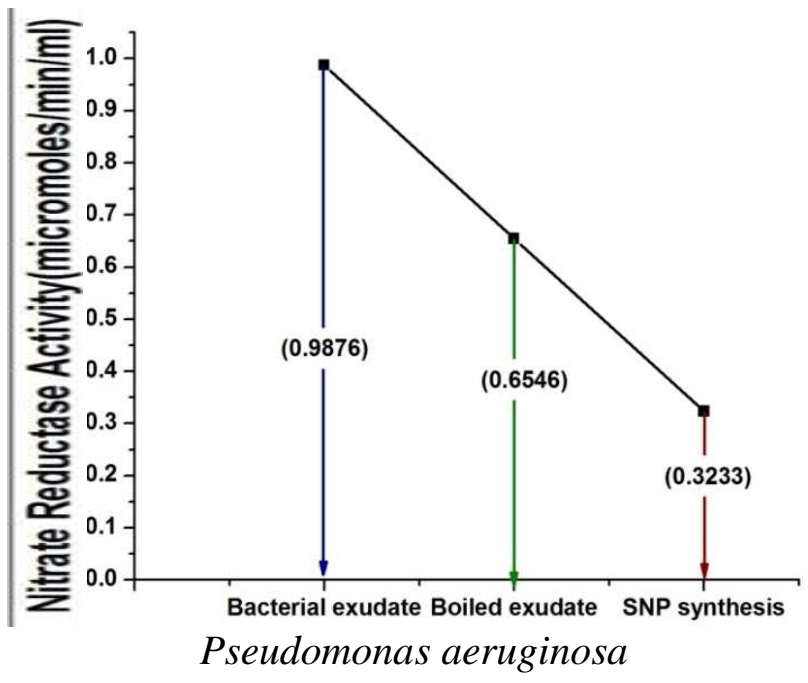


Fig.6 Antimicrobial activity of silver nanoparticles against various pathogenic bacterial strains. (a)Pseudomonas aeruginosa (b)Staphylococcus aureus \& (c) Escherichia coli shown by welldiffusion method.
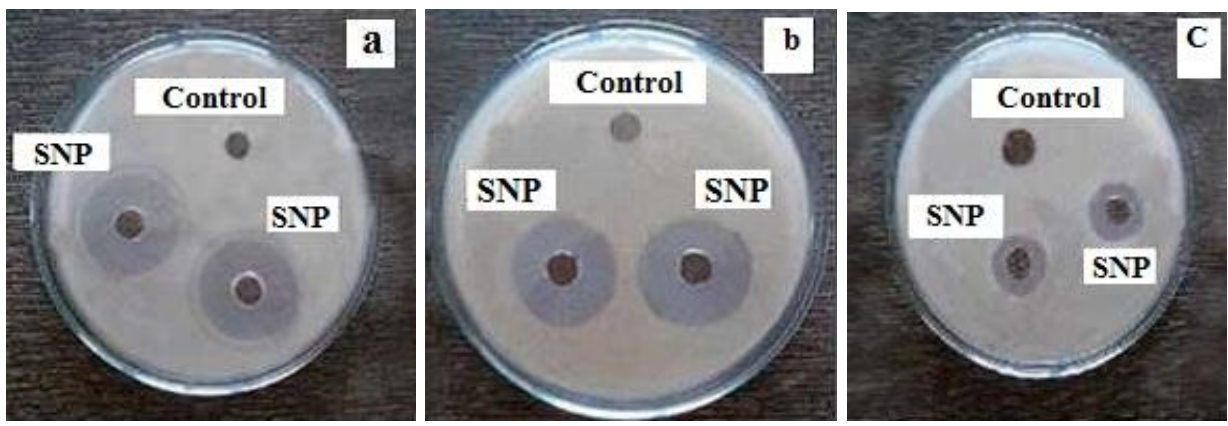

In the present study, biosynthesis of AgNPs by Pseudomonas aeruginosa was observed by the colour change of culture filtrate from yellow to brown, after addition of $1 \mathrm{mM}$ solution of silver nitrate. The change in colour clearly indicated that there was formation of AgNPs in the reaction mixture (Shanmugaiah et al., 2015). The UV-Vis spectrophotometer analysis revealed that the absorbance peaks at $\sim 435 \mathrm{~nm}$, which are very specific for the synthesis of AgNPs. The XRD pattern thus obtained clearly shows (111), (200), (220), and (311) planes, and exhibit that the synthesized AgNPs by the Pseudomonas aeruginosa were crystalline in nature (Konish et al., 2004).

The results of TEM analysis clearly showed that bacteria have the potential to form smaller size particles with higher surface area. (Sukanya et al., 2013; Manivasagan et al., 2013; Birla et al., 2009). Antibacterial activity of biogenically synthesized AgNPs was evaluated against various pathogenic organisms such as Escherichia coli, Staphylococcus aureus and Pseudomonas aeruginosa using well-diffusion method. The AgNPs synthesized from Pseudomonas aeruginosa strain showed the highest antibacterial activity against Pseudomonas aeruginosa followed by Staphylococcus aureus and Escherichia coli (Birla et al., 2009). The thrust to develop eco-friendly procedures for the production of nanoparticles arises from the extremely recent nanotechnology research. Extracellular biosynthesis of silver nanoparticles was achieved by an easy biological procedure using Pseudomonas aeruginosa as the reducing agent. The method exploits a cheap and easily available biomaterial not explored so far for the synthesis of metallic nanoparticles.

In conclusion, biological synthesis of metal nanoparticles using bacteria is a reliable and with ecofriendly protocol Bacteria. The mechanism of synthesis of metal nanoparticles by microbes is not clearly explored. Pseudomonas aeruginosa was capable of producing silver nanoparticles extracellularly and were quite stable in the solution .Applications of such eco-friendly nanoparticles in bactericidal applications, makes this method potentially exciting for the large-scale synthesis of other nanomaterials.

\section{Acknowledgement}

Sincerely thanks to Marine Microbiology $\mathrm{Lab}$ at the National Institute of Oceanography and Fisheries, Alexandria, Egypt (NIOF). The author thanks his respective managements for encouragement, facilities and support. 


\section{References}

Ahmad, A., S. Senapati, M.I .Khan, R. Kumar, M. Sastry 2005. J. Biomed. Nanotechnol., 1: 47.

Beveridge, T.J., M.N. Hughes, H. Lee, K.T. Leung, R.K., Poole, I. Savvaidis,et al, Adv. Microb. Physiol., 38: 177.

Birla, S.S., Tiwari, V.V., Gade, A.K., Ingle, A.P., et al. 2009. Fabrication of silver nanoparticles by Phoma glomerata and its combined effect against Escherichia coli, Pseudomonas aeruginosa and Staphylococcus aureus. Lett. Appl. Microbiol., 48: 173-179.

Bruins, R.M., S. Kapil, S.W. Oehme, Ecotoxicol. Environ. Saf., 45: 198.

Duran, N., G.I.H. De Souza, O.L. Alves, E. Esposito, P.D. Marcato, J. Nanotechnol., 122.

Gupta, A., Bonde, S.R., Gaikwad, S., Ingle, A., et al. 2014. Lawsonia inermismediated synthesis of silver nanoparticles: activity against human pathogenic fungi and bacteria with special reference to formulation of an antimicrobial nanogel. IET Nanobiotechnol., 8: 172-178.

Karthik, L., Kumar, G., Vishnu Kirthi, A., Rahuman A.A., et al. 2014. Streptomyces sp. LK3 mediated synthesis of silver nanoparticles and its biomedical application. Bioprocess Biosyst. Eng., 37: 261-267.

Konish, Y., Deshmukh, N., Tsukiyama, T., Saitoh, N. 2004. Microbial preparation of gold Nanoparticles by anaerobic bacterium. Trans. Mater. Res. Soc. Jpn. 29: 2341-2343.

Manivasagan, P., Venkatesan, J., Senthilkumar, K., Sivakumar, K., et al., 2013. Biosynthesis, antimicrobial and cytotoxic effect of silver nanoparticles using a novel Nocardiopsis sp. MBRC1. BioMed. Res. Int., 1-9.

Mewada, A., G. Oza, S. Pandey, Madhuri Sharon 2012. J. Microbiol. Biotechnlol. Res., 2(3)in press.
Priyaragini, S., Sathishkumar, S.R., Bhaskararao, K.V., 2013. Biosynthesis of silver nanoparticles using actinobacteria and evaluating its antimicrobial and cytotoxicity activity. Int. J. Pharm. Sci., 5: 709-712.

Rai, M., Birla, S., Ingle, A.P., Gupta, I., et al., 2014. Nanosilver: an inorganic nanoparticle with myriad potential applications. Nanotechnol. Rev., 3: 281309.

Rai, M., Ingle, A.P., Gade, A.K., Duarte, M.C.T., et al., 2015. Three Phoma spp. synthesised novel silver nanoparticles that possess excellent antimicrobial efficacy. IET Nanobiotechnol., 9: 280287.

Saitou, N., Nei, M. 1987. The neighborjoining method: a new method for constructing phylogenetic trees. Mol. Biol. Evol., 4: 4064-4025.

Sastry, M,. Ahmad, A., Khan, M.I. \& Kumar, R. 2003. Biosynthesis of metal nanoparticles using fungi and actinomycetes, Curr. Sci., 85: 162-170.

Sastry, M., A. Ahmad, M.I. Khan, R. Kumar Curr. Sci., 85: 162.

Shah, R., G. Oza, S. Pandey, Madhuri Sharon. J. Microbiol. Biotechn. Res., 2(3)in press.

Shanmugaiah, V., Harikrishnan, H., Al-Harbi, N.S., Shine, K., et al., 2015. Facile synthesis of silver nanoparticles using Streptomyces sp.VSMGT1014 and their antimicrobial efficiency. Dig. J. Nanomater. Bios., 10: 179-187.

Sukanya, M.K., Saju, K.A., Praseetha, P.K., Sakthivel, G., 2013. Therapeutic potential of biologically reduced silver nanoparticles from actinomycete cultures. J. Nanosci., 1-8.

Tillmann, P. 2004. Stability of silver nanoparticles in aqueous and organic media. J. Mater. Chem., 140-146.

Tsibakhashvili, N.Y., Kirkesali, E.I., Pataraya, D.T., Gurielidze, M.A., et al., 2011. Microbial synthesis of silver nanoparticles by Streptomyces glaucus and Spirulina platensis. Nanomater., 2, 
306-310.

Udaya Prakash, N.K., Bhuvaneswari, S., Prabha, S.B., Kavitha, K., et al., 2014. Green synthesis of silver nanoparticles using airborne Actinomycetes. Int. J Chem. Tech. Res., 6: 4123-4127.

Vighneshwaan, N., Kathe, A.A., Vardarajan,
P.V., Nachane, R.P., Bala Subramanian, R.H. 2007. A simple route for the synthesis of silverprotein (core-shell) Nanoparticles using spent mushroom substrate (SMS). Langmuir, 23: 71137117.

\section{How to cite this article:}

Hala Ezzat Abou El-Hassayeb. 2016. Bactericidal Application of Biosynthesized Silver Nanoparticles. Int.J.Curr.Microbiol.App.Sci. 5(7): 785-793.

doi: http://dx.doi.org/10.20546/ijcmas.2016.507.090 